Signal \& Image Processing : An International Journal (SIPIJ) Vol.6, No.3, June 2015

\title{
DESIGN REALIZATION AND PERFORMANCE EVALUATION OF AN ACOUSTIC ECHO CANCELLATION (AEC) IN HANDS-FREE COMMUNICATIONS USING RECURSIVE ADAPTIVE ALGORITHM
}

\author{
Azeddine Wahbi ${ }^{1}$, Ahmed Roukhe ${ }^{2}$ and Laamari Hlou ${ }^{1}$ \\ ${ }^{1}$ Laboratory of Electrical Engineering and Energy System Faculty of Science, University \\ Ibn Tofail, B.P. 133. Kenitra, Morocco \\ ${ }^{2}$ Laboratory of Atomic, Mechanical, Photonics and Energy Faculty of Science, \\ University Moulay Ismail, B.P. 11201. Meknes, Morocco
}

\begin{abstract}
Nowadays, in the field of communications, AEC (acoustic echo cancellation) is truly essential with respect to the quality of multimedia transmission. In this paper, we designed and developed an efficient AEC based on adaptive filters to improve quality of service in telecommunications against the phenomena of acoustic echo, which is indeed a problem in hands-free communications.

The main advantage of the proposed algorithm is its capacity of tracking non-stationary signals such as acoustic echo. In this work the acoustic echo cancellation (AEC) is modeled using a digital signal processing technique especially Simulink Blocksets. The algorithm's code is generated in Matlab Simulink programming environment. At simulation level, results of simulink implementation prove that module behavior is realistic when it comes to cancellation of echo in hands free communication using adaptive algorithm.
\end{abstract}

Results obtained with our algorithm in terms of ERLE criteria are confronted to IUT-T recommendation G.168.

\section{KEYWORDS}

Digital Signal Processing (DSP), Adaptive Algorithm, Acoustic Echo Cancellation (AEC), hands-free communications, Echo Return Loss Enhancements (ERLE).

\section{INTRODUCTION}

Acoustic echo occur when an audio signal is reverberated in a real environment, resulting in the original intended signal plus attenuated and delay time. Also Hands-free communications are frequently, an interesting challenge, because the interference of echo in speech causes usually a problem in communications [1]. It can lead to an unwanted distortion in the quality of 
Signal \& Image Processing : An International Journal (SIPIJ) Vol.6, No.3, June 2015

communication. However, an acoustic echo cancellation must be strictly implemented to preserve this quality.

Recently, adaptive filtering technique has emerged as an important technology towards hands free communications. It's used to design the AEC for enhancing the quality of speech.

Different works of RLS adaptive algorithm for echo cancellation are presented [2-3-4-5-6]. In this work the acoustic echo cancellation (AEC) is modeled in Simulink using digital filters especially adaptive Recursive Least Square (RLS) Algorithm.

The recursive algorithm has the best capacity when tracking non-stationary signals, such as speech or sound, but has also a very high convergence rate with high computational cost, compared to the NLMS algorithm modeled by A. Wahbi in [7].This algorithm has a low complexity, but it is robust for both stationary as well as non stationary environment.

The paper is structured as follows: section II presents digital adaptive filters for echo cancelling, section III presents simulation results and Section IV concludes this paper.

\section{Digital AdAPTIVE FiLTERS FOR ECHO CANCELLING}

\subsection{Adaptive Filters}

Adaptive filters can be classified into two great categories based on their impulse response:

- Adaptive finite impulse response filters;

- Adaptive infinite impulse response filters [8-9-10].

The adaptive filter considered in this work is considered to be finite impulse response (FIR) category with $\mathrm{N}$ coefficients, such that the output of the adaptive filter can be expressed as:

$$
y(n)=\sum_{k=0}^{N-1} h_{k}(n) x(n-k)
$$

Adaptive FIR filters have many structures, such as the adaptive transversal filter, the network preacher, the systolic array, etc.

The structure of adaptive transversal filters, which are the subject of our primary concern regarding echo cancellation, as shown in Figure 1. 
Signal \& Image Processing : An International Journal (SIPIJ) Vol.6, No.3, June 2015

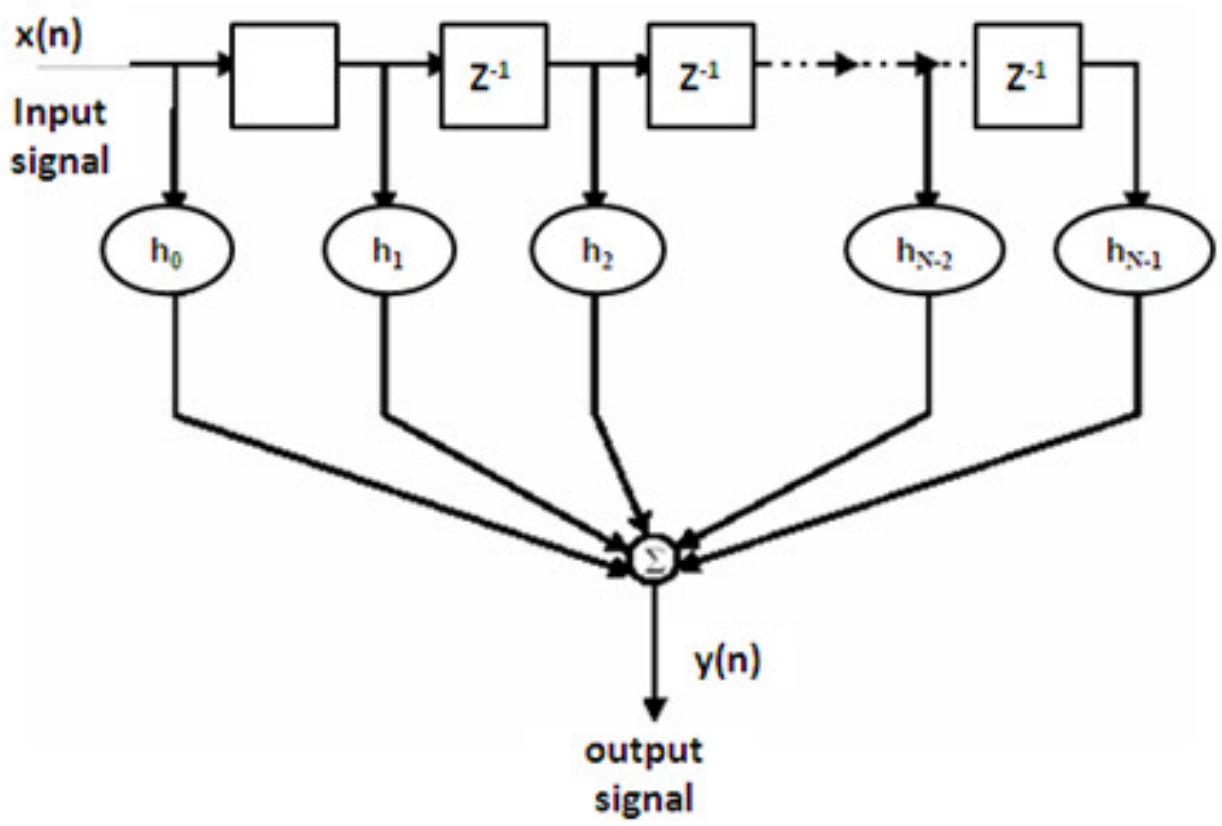

Figure 1. Finite Impulse response (FIR) filter structure.

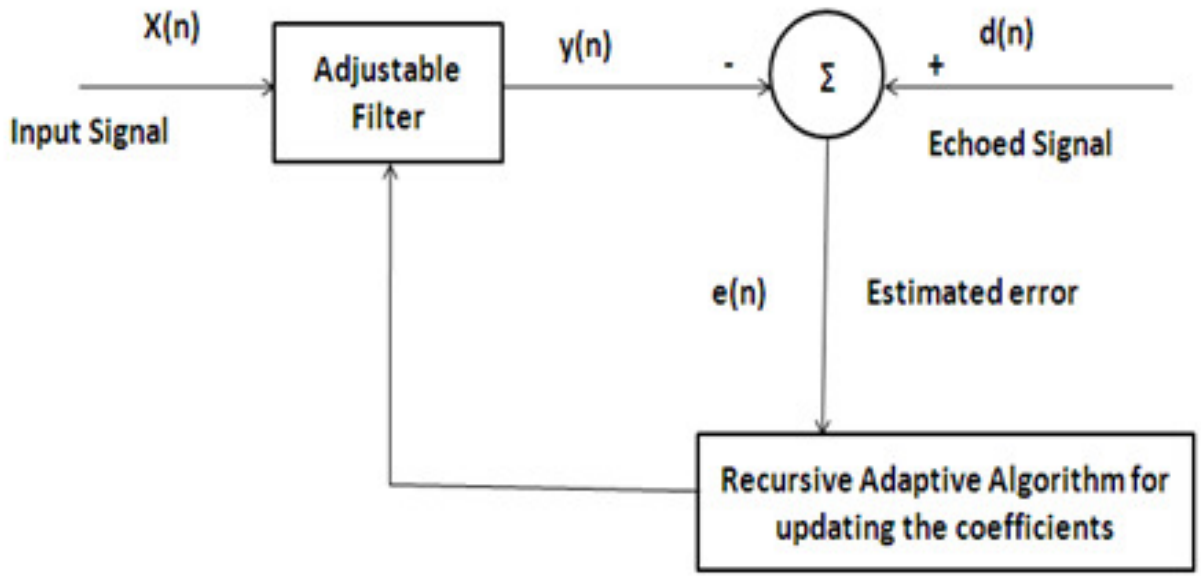

Figure 2. Adaptive filter structure

\subsection{Adaptive Echo Cancellation}

One of the adaptive filter applications is the adaptive echo canceller. Figure 2 and Figure 3 describe its structure where the desired response is composed of an original signal plus the echoed, which is uncorrelated with the signal.

The filter input is a sequence of an echoed signal which is correlated with the echoed signal in the desired signal.

By using the RLS algorithm inside the adaptive filter, the error term e(n) produced by this system is then the original signal with the echoed signal cancelled [11]. 


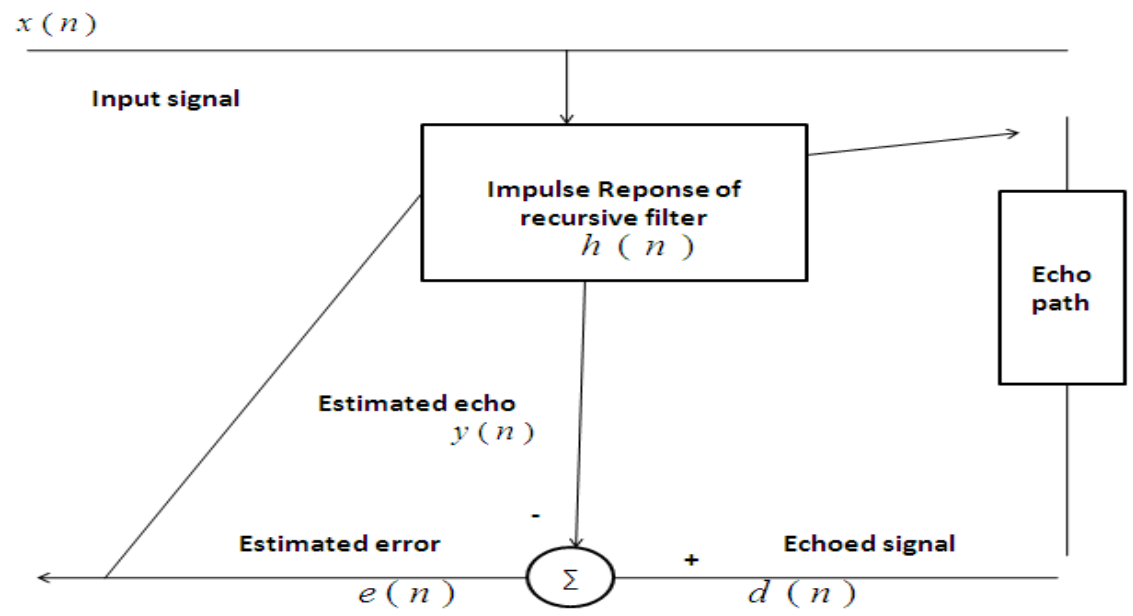

Figure 3. Block diagram of the acoustic echo canceller

\subsection{RLS Algorithm}

Updating the coefficients of a recursive adaptive filter requires the minimization of a cost function; it's defined by the following equation:

$$
C(n)=\sum_{K=0}^{n} \lambda^{n-k} e_{n}^{2}(k)
$$

Where, $0<\lambda<1$ represents the forgetting factor, which gives more importance to the most recent samples. But, the more distant time samples are omitted.

Generally, an adaptive filter uses two inputs: Input signal and desired signal. However, the input signal is defined as the signal to be processed. But, the desired signal is given to the adaptive filtering, so it has the original signal.

The Output port outputs the enhanced signal, which can be sample or frame based. The error signal or estimated signal is very useful for updating the adaptive filter.

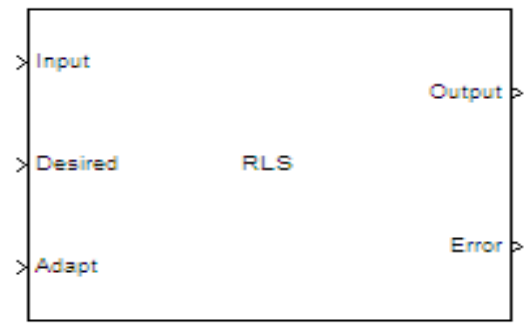

Figure 4. RLS Filter block [12]

Hence, we define the primary input signal to be a delayed version of the measurement $y(n)$ and the reference signal to be the measurement itself.

We then apply the following RLS algorithm which Tracks the desired solution. 
Signal \& Image Processing : An International Journal (SIPIJ) Vol.6, No.3, June 2015

$$
\begin{aligned}
& y(n-1)=h(n-1) x(n) \\
& e(n-1)=d(n)-y(n-1) \\
& p_{\mu}=x_{n}^{T} \theta x_{n}
\end{aligned}
$$

Where

$$
\begin{gathered}
\theta=\left(\begin{array}{ccccc}
\mu^{n-1} & 0 & 0 & \mathrm{~L} & 0 \\
0 & \mu^{n-2} & 0 & \mathrm{~L} & 0 \\
0 & 0 & \mu^{n-3} & \mathrm{~L} & 0 \\
\mathrm{~L} & \mathrm{~L} & \mathrm{~L} & \mathrm{~L} & \mathrm{~L} \\
0 & 0 & 0 & \mathrm{~L} & 1
\end{array}\right) \\
=\mu^{-1} p_{\mu}^{-1}(n-1)-\frac{\mu^{-2} p_{\mu}{ }^{-1}(n-1) x_{n} x_{n}{ }^{T} p_{\mu}{ }^{-1}(n-1)}{1+\mu^{-1} x_{n} p_{\mu}{ }^{-1}(n-1) x_{n}{ }^{T}} \\
=\lambda^{-1} p_{\mu}(n-1)-x_{n} x_{n}{ }^{-1}(n-1)-k(n) x_{n}{ }^{T} p_{\mu}{ }^{-1}(n-1) \\
k(n)=\frac{\mu^{-1} p_{\mu}{ }^{-1}(n-1) x_{n}}{1+\mu^{-1} x_{n}^{T} p_{\mu}{ }^{-1}(n-1) x_{n}}=p_{\mu}{ }^{-1}(n) x_{n} \\
h(n)=h(n-1)+k^{T}(n) e(n)
\end{gathered}
$$

Where $\mu^{-1}$ denotes the reciprocal of the exponential weighting factor. The variables are as follows.

Table 1. Table Variable of RLS Algorithm

\begin{tabular}{|l|l|}
\hline Variable & Description \\
\hline $\mathrm{n}$ & The present step of the algorithm \\
\hline $\mathrm{x}(\mathrm{n})$ & The input at step $\mathrm{n}$ \\
\hline $\mathrm{h}(\mathrm{n})$ & The adaptive filter coefficients at step $\mathrm{n}$ \\
\hline $\mathrm{P}(\mathrm{n})$ & The inverse correlation matrix at step $\mathrm{n}$ \\
\hline $\mathrm{k}(\mathrm{n})$ & The gain vector at step $\mathrm{n}$ \\
\hline $\mathrm{y}(\mathrm{n})$ & The estimated echo at step $\mathrm{n}$ \\
\hline $\mathrm{e}(\mathrm{n})$ & The estimated error at step $\mathrm{n}$ \\
\hline $\mathrm{d}(\mathrm{n})$ & The echoed signal at step $\mathrm{n}$ \\
\hline$\mu$ & The Variable Forgetting Factor to adjust. Where $0<\mu<1$ \\
\hline
\end{tabular}


Signal \& Image Processing : An International Journal (SIPIJ) Vol.6, No.3, June 2015

\subsection{Flow chart of RLS algorithm}

Flow chart in Figure 5 describes the flow of recursive least square algorithm.

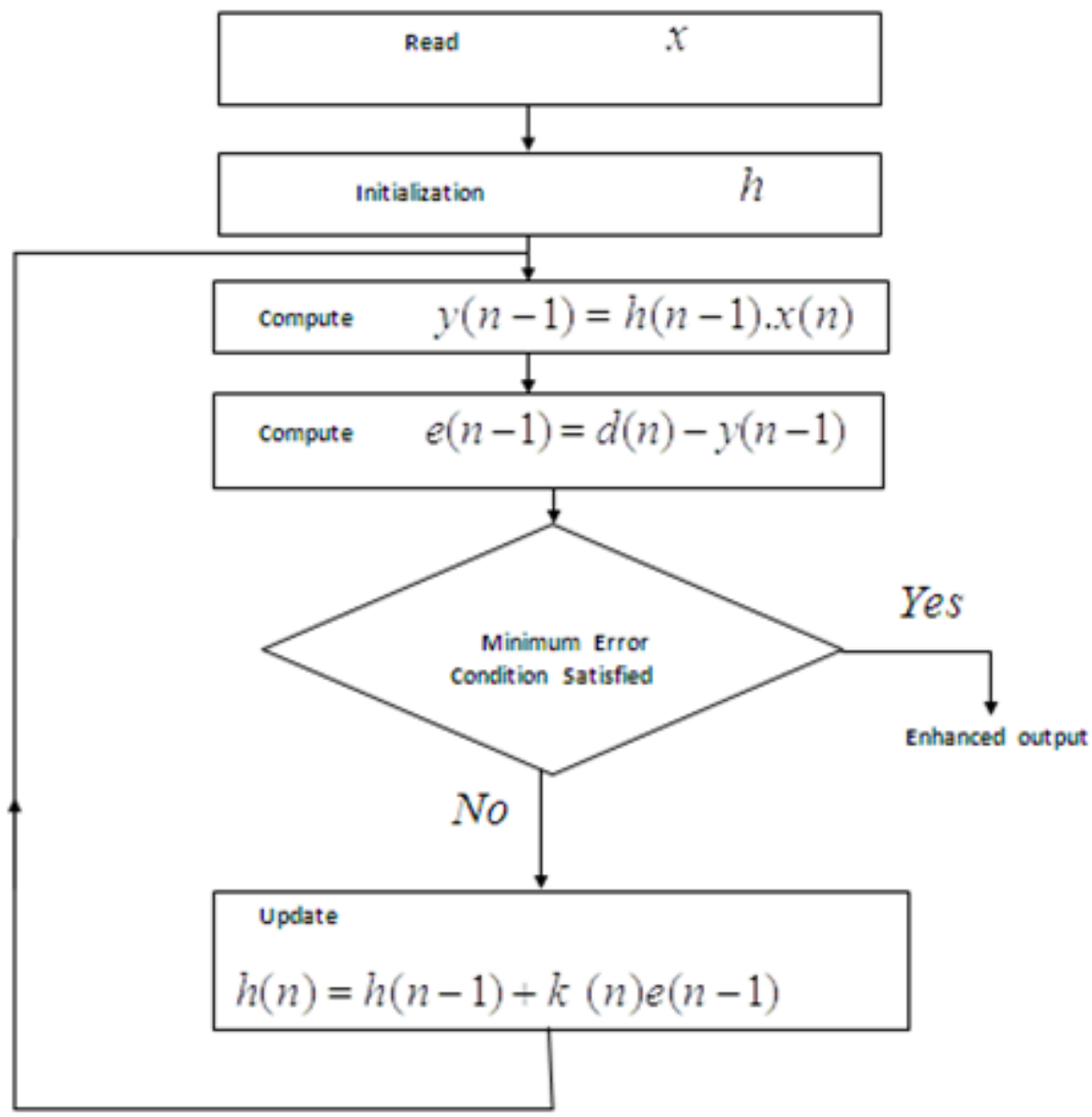

Figure 5. Flow chart of recursive adaptive algorithm

\subsection{Echo Return Loss Enhancements (ERLE)}

The robustness of the proposed AEC is verified by using Echo Return Loss Enhancements (ERLE) criteria. It is calculated as:

$$
\begin{aligned}
& \text { ERLE } E_{d B}=10 \log _{10}\left(\frac{\text { Power }(\text { Echoed signal })}{\text { Power }(\text { Output AEC })}\right. \\
& \quad=\text { Power }(\text { Echoed signal })_{d B}-\text { Power }(\text { Output AEC })_{d B}
\end{aligned}
$$

However, the ERLE plays an important role in digital signal processing; it measures the attenuation of the echo signal at the output of an AEC [13-14]. 
Signal \& Image Processing : An International Journal (SIPIJ) Vol.6, No.3, June 2015

\section{SIMULATION RESULTS}

\subsection{Echo Canceller Modeling using Simulink Environment}

In this work we designed the model using Simulink Blockset. For the input signal we used an audio data in wav format, $8000 \mathrm{~Hz}$ sampling rate.

The delay of time is approximately $30 \mathrm{~ms}$ and the length is 256 samples. AEC implementation is setup with RLS adaptive filter of length 32. In addition, the forgetting factor is chosen as $\mu=0.5$.

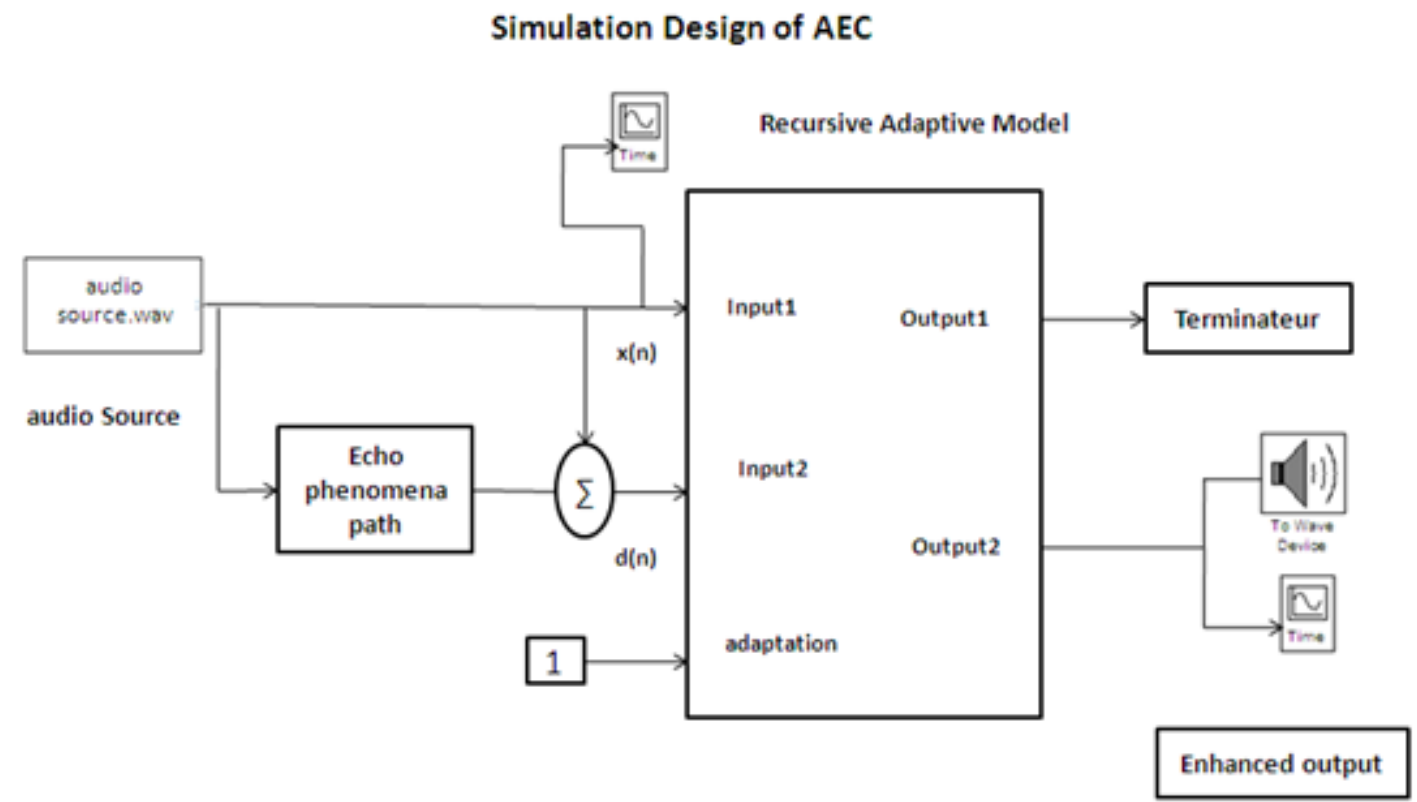

Figure 6. Acoustic Echo Cancelation under simulink

\subsection{Simulink Results}

The original speech signal, echoed signal and enhanced signal are shown in Figures 7, 8 and 9 respectively. In this case, we observe the input signal (the original signal with echo) and how this echo is removed from the original signal after crossing by the "recursive adaptive AEC", knowing the echo signal has less amplitude than the original signal. Whereas, the results, demonstrating how the signal is filtered, and the result is an output signal with less amplitude than the input signal and without echo. 
Signal \& Image Processing : An International Journal (SIPIJ) Vol.6, No.3, June 2015

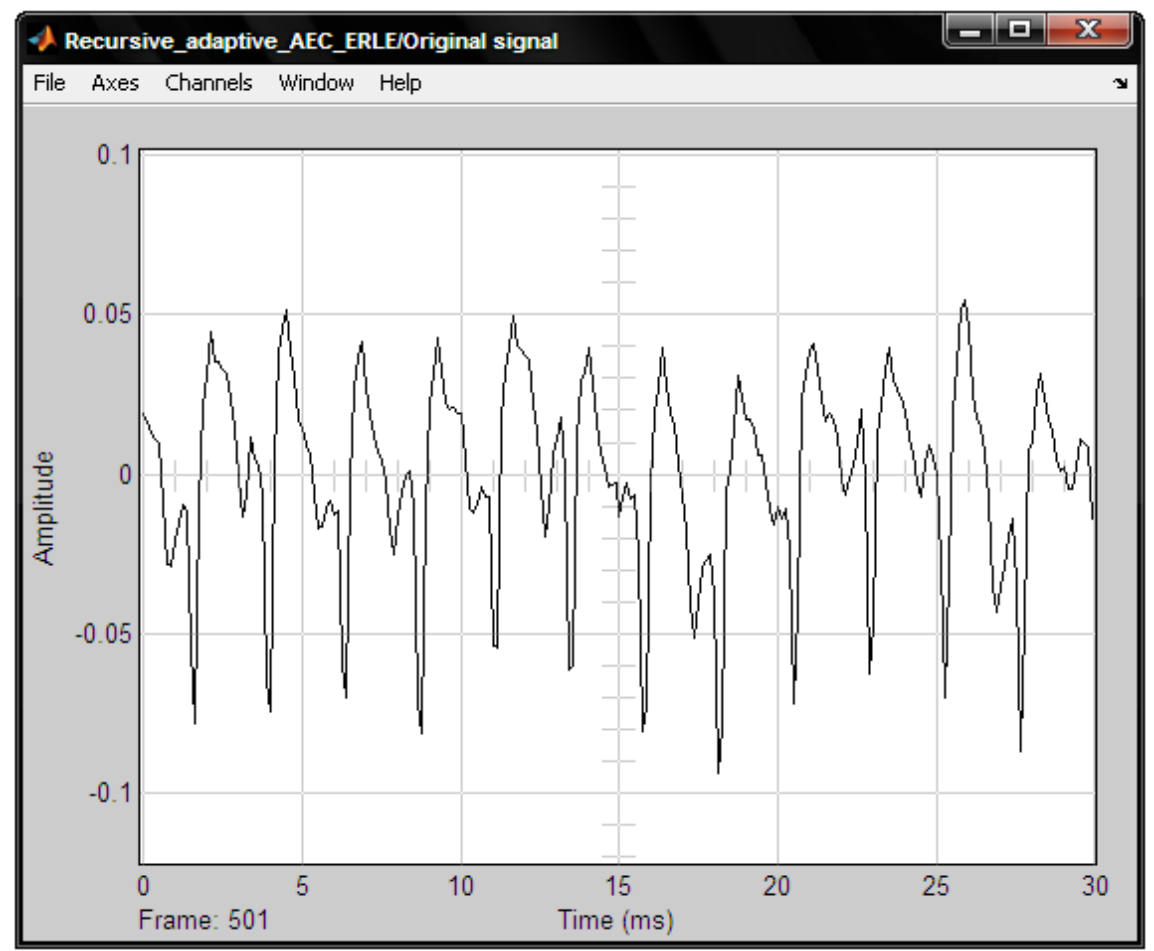

Figure 7. Result obtained using Simulink simulation (Original)

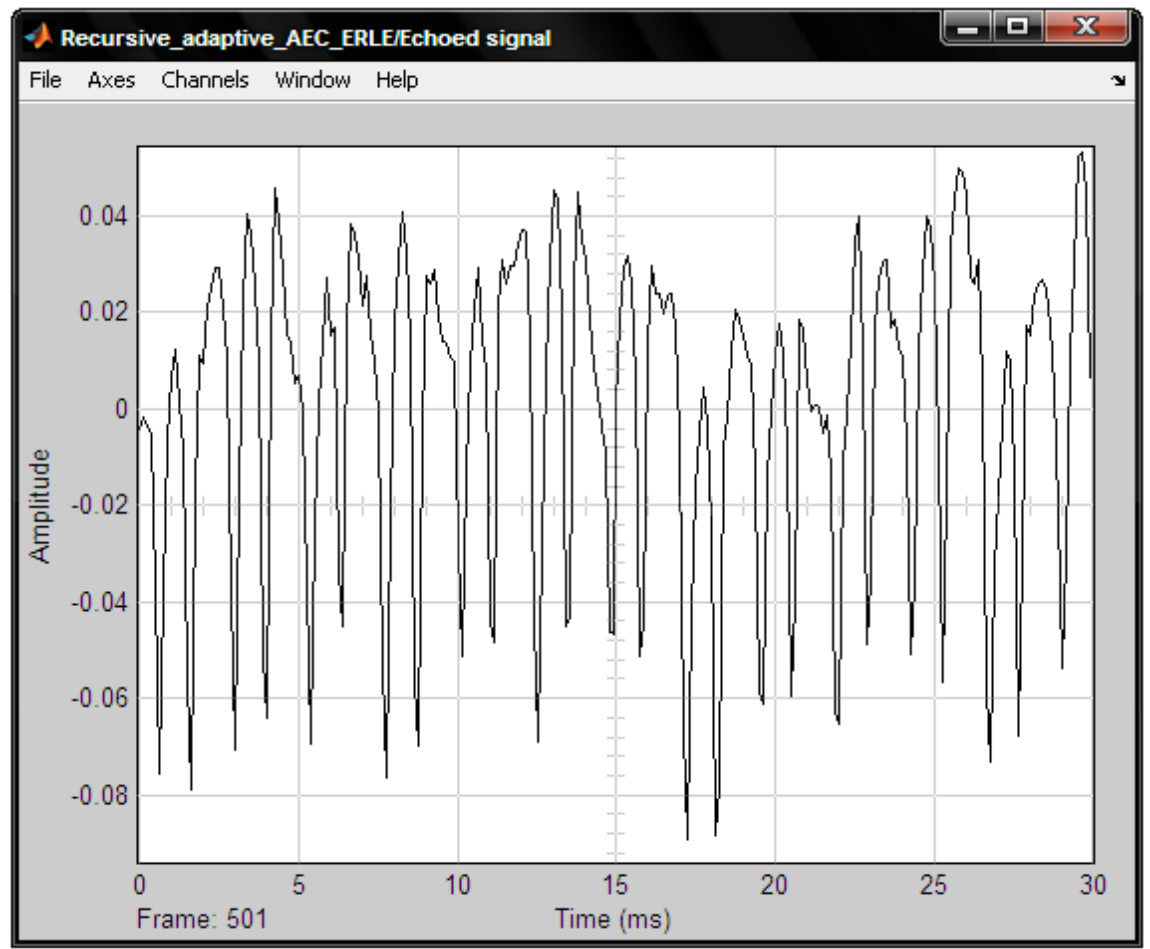

Figure 8. Result obtained using Simulink simulation (Echoed signal) 
Signal \& Image Processing : An International Journal (SIPIJ) Vol.6, No.3, June 2015

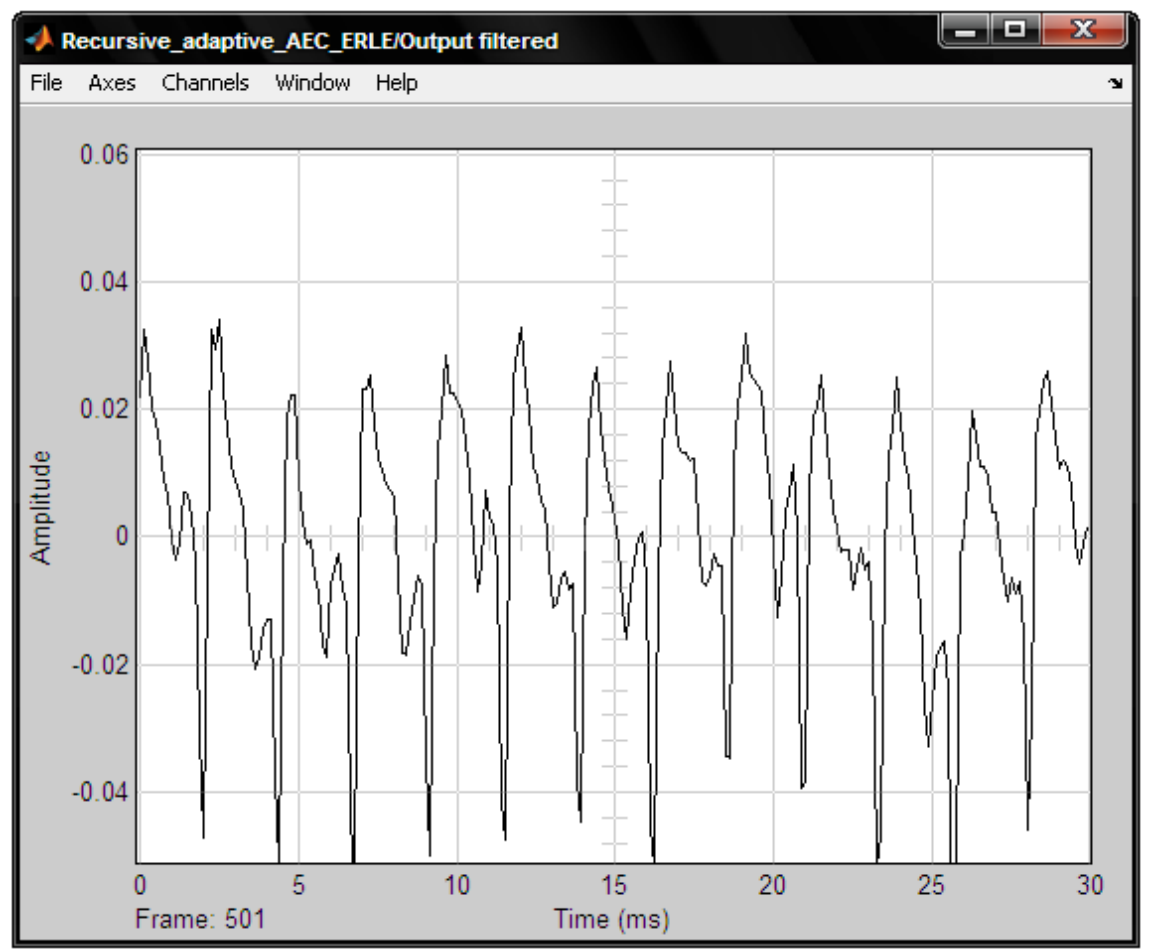

Figure 9. Result obtained using Simulink simulation (filtered output)

Table 2. ERLE Improvement of AEC

\begin{tabular}{|c|c|}
\hline Time (S) & ERLE of filtered output (dB) \\
\hline 3 & 38.777 \\
\hline 6 & 39.69 \\
\hline 9 & 33.53 \\
\hline 12 & 32.18 \\
\hline 15 & 41.34 \\
\hline 18 & 31.31 \\
\hline 21 & 34.84 \\
\hline 24 & 25.9 \\
\hline 27 & 40.89 \\
\hline 30 & 39.46 \\
\hline
\end{tabular}

The convergence rate of the AEC to find the optimal value of the RLS filter to cancel the echo depends on various factors such as the forgetting factor parameter. In this case, we modified the step-size, the filter length and the number of samples to better test the performance of this AEC against acoustic echo. We also noticed that the smaller the step size is then AEC converges to the optimal value when increasing of ERLE value.

The performances of the AEC based on the adaptive algorithm can be evaluated using the ERLE criteria. The obtained results described in table II prove the efficiency of the proposed AEC 
Signal \& Image Processing : An International Journal (SIPIJ) Vol.6, No.3, June 2015

based RLS algorithm against the phenomena working in time changing environments as acoustic echo does.

\section{CONCLUSION}

In this paper, a module consisting of an AEC based recursive least square adaptive algorithm is proposed for improving the quality of the speech signal in hands-free communications systems. Effectiveness of Proposed AEC based recursive algorithm has been tested using ERLE criteria. It concludes that our approach shows more efficiency in terms of capacity of tracking nonstationary signals and convergence.

In subsequent works, we will implement this module in real time onboard an autonomous DSK C6713, thanks to its low computational cost and its user-friendly Simulink programming implementation. Also we will improve the performances of the proposed module by using another approaches based digital signal processing for developing a new AEC with higher convergence rate and lesser computational complexity.

\section{REFERENCES}

[1] Stefan Goetze et all, "Hands-Free Telecommunication for Elderly Persons Suffering from Hearing Deficiencies", IEEE Healthcom, 2010, pp 209-2014.

[2] S.Dhamija et al, "Adaptive filtering algorithms for channel equalization and echo cancellation", IJCTA, Vol 2 (5), 2011, pp 1577-1581.

[3] Raghava Yathiraju, "Acoustic Echo Cancellation Using Conventional Adaptive Algorithms", International Journal of Inventive Engineering and Sciences (IJIES), Volume-1, Issue-12, November 2013, PP 18-24.

[4] A. Munjal et all, "RLS Algorithm For Acoustic Echo Cancellation", Proceedings of 2nd National Conference on Challenges \& Opportunities in Information Technology (COIT), 2008.

[5] P.Rajesh, A.Sumalatha, "A Novel Approach of Acoustic Echo Cancellation Using Adaptive Filtering", International Journal of Engineering Research \& Technology (IJERT), Vol. 1 Issue 5, 2012, pp 1-10.

[6] Arvind Kourav and Binod K. Soni, "RLS Algorithm for Adaptive Echo Cancellation", International Journal on Emerging Technologies, Vol. 2 Issue 1, 2011, pp 35-38.

[7] A.Wahbi et all, "Real-Time acoustic echo cancellation for hand-free communication by implementing a LMS algorithm onboard an autonomous DSK C6713 ", 3rd International Conference on Systems, Modeling and Design, Kenitra (MIC-SMD 2013), 2013

[8] H. Sayed, "Fundamentals of Adaptive Filtering", Wiley, 2003.

[9] R. Gnanasekaran et all, "A note on block implementation of IIR digital filters", Proc. IEEE, vol. 65, pp. 1063-1064, July 1977.

[10] Yen-Liang Chen et all, "A universal look-ahead algorithm for pipelining IIR filters", IEEE VLSIDAT'08, pp. 259-262, Apr. 23-25, 2008.

[11] Woon-Seng Gan et Sen M. Kuo. 2007. Embedded Signal Processing with the Micro Signal Architecture in Wiley.

[12] The Mathworks Inc., Matlab and Simulink User's Guide, 2012

[13] F. Albu, "Improved variable Forgetting factor recursive least square algorithm ", Control Automation Robotics \& Vision (ICARCV), 12th International Conference on , 2012, pp 1789-1793.

[14] C. Stanciu et all, "Variable-forgetting factor RLS for stereophonic acoustic echo cancellation with widely Linear model", 20 th European Signal processing Conference (EUSIPCO), 2012. 